der einzige Beurteilungsfaktor ist. Man kann auch aus den anderen Analysenwerten insbesondere aus Säure und zuckerfreiem Extrakt Schlüsse dahin ziehen, ob ein gespriteter Most vorliegt oder nicht. Von besonderem Werte ist auch die Zungenprobe. Es ist bei einiger Übung durchaus nicht sehwer schon hierdurch festzustellen, ob ein gespriteter Most oder ein vergorenes $\mathrm{Er}$ zeugnis in Frage kommt.

Dr. Große-Bohle: Bei der Sache selbst müssen wir anch die Absicht des Gesetzgebers in Betracht ziehen. Ich glaube, es war nicht seine Absicht, die Einfuhr dieser Weine zu verbieten.

Der Vorsitzende: Es hat sich niemand mehr zum Wort gemeldet. Ich schließe deshalb die Diskussion. Jedenfalls haben die Verhandlungen gezeigt, daß Herr Prof. Fresenius in seinem Vortrag ein Thema erörtert hat, das das größte Interesse seitens der Nahrungsmittelchemiker erheischt. Wir haben nicht die Absicht gehabt, die Frage bezüglich der Einfuhrfähigkeit dieser Weine heute zu einer Entscheidung zu bringen. Wir haben nur in eine Aussprache darüber treten wollen, und ich glaube, daß diese der Kommission, die uns im nächsten Jahre über die Untersuchungsmethoden und Beurteilung der Süßweine Bericht erstatten soll, wertvolle Anhaltspunkte gegeben hat.

Wir gehen nunmehr in unserer Tagesordnung weiter und ich erteile das Wort Herrn Dr. Tillmans.

\title{
Eine bequeme Ausführungsart der Storch'schen Reaktion.
}

Von

\section{Dr. J. Tillmans.}

Mitteilung aus der Chemisch-hygienischen Abteilung (Abteilungsvorsteher: Dr. J. Tillmans) des Städtischen Hygienischen Instituts in Frankfurt a. M. (Direktor: Prof. Dr. M. Neifzer).

Die von Stor $\mathrm{ch}^{\mathbf{1}}$ ) angegebene Reaktion zum Nachweis von ungekochter Milch in gekochter Milch leidet bekauntlich an dem technischen Mangel, daß die vorgeschriebenen, sehr verdünnten Lösungen wenig haltbar sind. Aus diesem Grunde hat $\mathrm{Utz}^{2}$ ) im Jahre 1902 die Lösungen zu umgehen versucht, indem er das von ihm verwendete p-Phenylendiamin (Ursol D) in fester Form anzuwenden sich benühte. Da Utz der Ansicht war, daß bei der Reaktion, um keine Täuschungen aufkommen zu lassen, die geringe Menge p-Phenylendiamin angewendet werden müsse, wie sie in den dünnen Lösungen enthalten ist, so ließ er Tabletten mit einem indifferenten Stoff (Milchzucker), von denen jede gerade die betreffende Menge p-Phenylendiamin enthielt, herstellen. Im folgenden Jahre versuchte $\mathrm{Utz}^{3}$ ) in derselben Weise das Wasserstoffsuperoxyd durch einen festen Stoff zu ersetzen. Er schlug verschiedene Körper vor, unter ihnen auch eine Mischung von Bariumsuperoxyd mit Kaliumbisulfat. Auch hier glaubte Utz die Wasserstoffsuperoxydmenge, wie sie in den dünnen Lösungen angewandt wird, nicht überschreiten zu dürfen; er wandte deshalb diese Mischung ebenfalls in Tablettenform an.

Siegfeld ${ }^{4}$ ) hat die von $\mathrm{Utz}$ vorgeschlagenen Ersatzmittel nachgeprüft und gefunden, daß die Mischung von Bariumsuperoxyd und Kaliumbisulfat nicht brauchbar war, was nach Siegfeld's Ansicht auf der Säurewirkung auf das Enzym beruht.

1) 40. Bericht d. Laboratoriums f. landwirtschaftl. Versuche der Kgl. tierärztl. u. landwirtschaftl. Hochschule Kopenhagen 1898.

2) Chem.Ztg. 1902, 26,1121 .

3) Chem.Ztg. 1903, 27, 300.

4) Zeitschr. angew. Chem. 1908, 16, 770 . 
Siegfeld findet ferner, daß man unbedenklich das 1,5\%-ige Wasserstoffsuperoxyd für die Ausführung der Reaktion anwenden kann.

Auf unserer Hauptversammlung im Jahre 1907 in Frankfurt hat GrosseBohle ${ }^{1}$ ) mitgeteilt, daß man nach seinen Befunden beliebige Mengen festes $p$-Phenylendiamin anwenden könne (1 Messerspitze voll). Ferner hält Grosse-Boble die Anwendung des stärkeren $3 \%$-igen Wasserstoffsuperoxyds für unbedenklich.

Für die Vorprüfung der Milch erschien es uns wichtig, eine möglichst einfache Reaktion zu besitzen, die man auch unbedenklich von Laien ausführea lassen kann. Zu dem Zweck versuchten wir ebenfalls, das Wasserstoffsuperoxyd durch einen festen Körper zu ersetzen.

Es erschien uns nicht unwahrseheinlich, daß bei Anwendung von Bariumsuperoxyd die in der Milch stets vorhandene geringe Säuremenge ausreichen könnte, die für die Ausführung der Reaktion nötigen Mengen von Wasserstoffsuperoxyd frei zu machen. Bei näherer Prüfung erwies sich das in der Tat als richtig. Es ist also bei Anwendung von Bariumsuperoxyd, um Wasserstoffsuperoxyd zu erhalten, nicht notwendig, gleichzeitig ein saueres Salz anzuwenden, wie Utz es getan hat, sondern man kann das. Freimachen des Wasserstoffsuperoxyds der in der Milch stets vorhandenen Milchsäure bezw. den saueren Salzen überlassen. Ebenso ist es auch nach unseren Befunden gleichgültig, welche Mengen von p-Phenylendiamin man anwendet. Ob dieser Widerspruch mit den Befunden von $\mathrm{U} t \mathrm{z}$ vielleicht daher rührt, daß dieser Körper jetzt in reinerer Form geliefert wird als früher, vermag ich nicht zu entscheiden.

Die Ausführung der Reaktion mit festem p-Phenylendiamin und Bariumsuperoxyd gestaltet sich außerordentlich einfach. Ich möchte Ihnen die Ausführung der Reaktion zeigen. Ich gieße in zwei Bechergläser je $10-20 \mathrm{ccm}$ ungekochte bezw. gekochte Milch ein. Aus diesen zwei Streugläsern, wie sie für Pfeffer und Salz benutzt werden, von denen das eine p-Phenylendiamin, das andere Bariumsuperoxyd enthält, streue ich nun in die Milch jedesmal eine Prise ein. Beim Umschätteln färbt sich dann die ungekochte Milch in wenigen Sekunden über Grün tiefblau, während die gekochte Mileh farblos bleibt. Von p-Phenylendiamin kann man, wenn die Färbung nicht sofort intensiv wird, eine oder zwei Prisen nachgeben, dagegen nimmt man am besten nur eine Prise Bariumsuperoxyd.

Bei Anwendung von viel Bariumsuperoxyd entsteht nämlich anstatt des blauen ein roter Farbstoff. Es rührt das daher, daß dann durch das viele Bariumsuperoxyd eine alkalische Reaktion in der Milch auftritt. Der betreffende Farbstoff ist aber in alkalischer Lösung bekanntlich rot. Ich kann Ihnen das in der Weise demonstrieren, daß ich nochmals zu dem Iuhalt des Gläschens, das den blauen Farbstoff enthält, mehrere Prisen Bariumsuperoxyd aufstreue. Nach einigem Umschütteln verwandelt sich dann der blaue Farbstoff in ein helles Rot. Betrachtet man also auch eine auftretende rote Färbung als Reaktion, so kann man auch so viel Bariumsuperoxyd einstreuen, wie man will. Verwendet man jedoch eine Prise auf $10-20 \mathrm{~cm}$ Milch, so wird der Farbstoff immer blau.

Da das p-Phenylendiamin dazu neigt, an der Wand zu kleben und sich schwer streuen läßt, so mischen wir es mit dem gleichen Volumen reinen Seesandes. Es läßt sich dann gut aus dem Streuglas ausschütteln. Bei Anwendung des festen p-Phenylendiamins ist es gleichgültig, ob man die freie Base oder das Chlorhydrat verwendet. Parallelversuche ergaben dieselbe Wirkung. Wir verwenden deshalb

1) Diese Zeitschrift 1907, 14, 78. 
p-Phenylendiamin pur. Merck, bei Bariumsuperoxyd wenden wir Barium peroxydatum hydricum pur. Merck an.

Auf die Dauer tritt allerdings auch bei gekochter Milch mach und nach eine Oxydation ein. Die ersten erkennbaren Spuren einer beginnenden Blaufärbung treten etwa 10 Minuten bis $1 / 4$ Stunde nach erfolgtem Zusatz ein. Nach 1 Stunde ist die Färbung immer noch eben erst erkennbar. Ganz anders bei ungekochter Milch. Die Reaktion tritt hier stets sofort ein. Wie Sie soeben gesehen haben, ist schon nach einigen Sekunden eine tiefblaue Färbung vorhanden. Es ist also ausgeschlossen, daß diese innerhalb weniger Sekunden entstehende Färbung mit den Spuren von Farbstoff, wie sie auch in gekochter Milch nach langer Zeit entstehen, verwechselt. werden könnte.

Wir haben auch versucht, um die Sache noch weiter zu vereinfachen, das Bariumsuperoxyd mit dem p-Phenylendiamin zu mischen, um so nur ein Streuglas notwendig zu haben. Es hat sich aber herausgestellt, daß derartige Gemische sich durch eine vor sich gehende Oxydation des p-Phenylendiamins nach und nach dunkel färben und an Wirksamkeit einbüßen. Man bleibt also am besten bei zwei Streugläsern, was ja auch die Handlichkeit des Verfahrens nicht nennenswert beeinträchtigen dürfte. Die Haltbarkeit der getrennt in den Streugläschen aufbewahrten Pulver läßt nichts zu wünschen übrig. Unsere in diesen Gläschen vorhandenen Pulver sind schon fast $1 / 2$ Jahr alt und haben ihre Wirksamkeit unveründert beibehalten.

Bei Mischungen von ungekochter und gekochter Milch ist noch bei einem Gehalt an ungekochter Milch von $5 \%$ nach 1 bis 2 Minuten eine sehr deutliche, unverkennbare Blaufärbung vorbanden. Auch bei einem Gebalt der Mischung von $2 \%$ ungekochter Milch ist nach wenigen Minuten eine schwache Blaufärbung zu bemerken.

Versuche mit ungekochter, gesäuerter Milch ergaben, daß die Reaktion mit festem p-Phenylendiamin and Bariumsuperoxyd erst bei hohen Säuregraden (etwa $50^{\circ}$ ) negativ wird. Ferner ergab sich in Ubereinstimmung mit den Beobachtungen anderer Autoren über die Peroxydase, daß ein Erhitzen auf $80^{\circ}$, eine Minute lang, die Reaktion negativ werden ließ, daß dagegen ein 5 Minuten langes Erhitzen auf $70^{\circ}$ nicht genügte, die Reaktion merklich abzuschwächen.

Ich hoffe, daß diese einfache Ausführungsart der Storch'schen Reaktion manchem ron Ihnen willkommen sein wird. Da jegliche Behandlung der Milch, jegliches Abmessen oder Pipettieren wegfüllt, so dürfte diese Art der Ausführung der Reaktion nicht nur im Laboratorium gute Dienste zu leisten vermögen; sie eignet sich vielmehr auch besonders zur Vorprüfung der Milch an Ort und Stelle durch Polizeibeamte, Laboratoriumsdiener und ähnliche mit der Vorprüfung der Nahrungsmittel betraute, nicht wissenschaftlich vorgebildete Personen.

Der Vorsitzende: Ich danke Herm Dr. Tillmans für seinen Vorirag und frage die Versammlung, $\mathrm{ob}$ sie eine Debatte wünscht.

\section{Diskussion:}

Dr. GroBe-Bohle: Teh möchte den Ferrn Referenten fragen, wie empfindlich die Reaktion ist.

Dr. Tillmans: Bei etwa $2 \%$ ungekochter Milch trat eine Blaufärbung ein, bei $5 \%$ eine ziemlich kräftige Blaufärbung. 
Der Vorsitzende: Wünscht noch einer der Herren das Wort? Das ist nicht der Fall. Wir gehen nunmehr zum letzten Punkt unserer heutigen Tagesordnung über: Zweite Beratung des Abschnittes Trinkbranntweine. - Referenten über die letzen Vereinbarungen mit den Interessenten sind die Herren K. von Buchka und A. Juckenack, von denen der erstere leider verhindert ist, heute hier anwesend zu sein.

\title{
Die Beurteilung der Trinkbranntweine.
}

\author{
Berichterstatter: Reg.-Rat Prof. Dr. A. Juckenack-Berlin.
}

M. H.! Herr Geheimer Oberregierungsrat Prof. Dr. K. v. Buchka, der die hier interessierenden Beurteilungsfragen seit der vorjährigen Versammlung in Kommissionssitzungen unter Zuziehung aller Interessentenkreise gründlich hat durchberaten lassen und ihnen fortgesetzt besondere Beachtung geschenkt bat, ist leider dienstlich verhindert, an den heutigen Beratungen teilnehmen zu können. Er hat mich daher gestern abend vor seiner Abreise gebeten, Ihnen einen kurzen Überblick über den bisherigen Verlauf der Angelegenheit zu geben.

Zunächst möchte ich hervorheben, daß infolge unserer Stellungnahme zur Beurteilung der Trinkbranntweine in Interessentenkreisen Vorwürfe gegen uns erhoben und Verdächtigungen uns gegenüber ausgesprochen worden sind, die vollständig an begründet sind und hier kurz zur Sprache gebracht werden müssen.

Besonders verletzend - ich will keinen schärferen Ausdruck gebrauchen, trotzdem er am Platze sein würde - ist die Verdächtigung, daß wir uns auf Veranlassung von Herrn Geheimrat Prof. Dr. Delbrü ck dazu hergäben, der Spirituszentrale Vorspanndienste zu leisten! Die ganze bisherige Entwickelung der Freien Vereinigung Deutscher Nahrungsmittelchemiker bürgt dafür, daß wir keiner Interessentengruppe Vorspanndienste leisten. Wir suchen als unabhängige Sachverständige lediglich der Allgemeinheit, dem öffentlichen Wohle zu dienen und die Gesetze im Sinne des Gesetzgebers auszulegen. Welche Entwickelung hat die Frage der Beurteilung der Trinkbranntweine tatsächlich genommen? Ganz unabhängig von unserer Vereinigung hat sich Herr Geheimrat Delbrü ck schon vor längerer Zeit - allerdings obne Erfolg an die zuständigen Reichsämter mit dem Ersuchen gewendet, eine reichsgesetzliche Regelung der Materie vorzubereiten und herbeizuführen. Inzwischen wurden in Interessentenkreisen Verhandlangen eingeleitet, die bezweckten, eine Konvention zwischen den Spiritusproduzenten, der Spirituszentrale und den Spiritusverbrauchern zustande zu bringen, die Vergünstigungen für die Destillationsbetriebe als Großabnehmer und dafür die Schaffung von Mindestgrenzen für den Alkoholgehalt der Trinkbranntweine vorsah. Gleichzeitig, aber ganz unabhängig von diesen Bestrebungen, wurde in unseren Kreisen über die Zunahme der Verwässerung der Trinkbranntweine geklagt und die Frage erörtert, ob es nicht zweckmäßig sei, das Kapitel „Trinkbranntwein" unserer alten "Vereinbarungen" neu zu heraten, da wir bekanntlich fortgesetzt neue Kapitel vornehmen und zwar in der Reihenfolge, die uns jeweilig nach Lage der im Verkehr beobachteten Verhälnisse zweckmäBig erscheint. Und wenn man z. B. in Berlin die Beobachtung macht, daß unter Namen wie "Nordhäuser", "Schlesischer" u. s. w Gemische an das Publikum abgegeben werden, die noch weniger als $20 \mathrm{Vol.}-\%$, ja gelegentlich nur 12 bis $13 \mathrm{Vol} .-0$ Alkohol enthalten, so ist es wahrlich für uns höchste Zeit, 